\title{
Comparison of different spectral cameras for image-guided organ transplantation
}

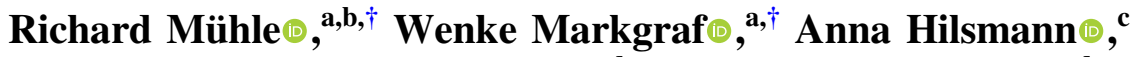 \\ Hagen Malberg, ${ }^{a}$ Peter Eisert $\odot,{ }^{c, d}$ and Eric L. Wisotzky ${ }^{c}$,d,* \\ ${ }^{a}$ Technische Universität Dresden, Institute of Biomedical Engineering, Dresden, \\ Germany \\ ${ }^{b}$ Technische Universität Dresden, Department of Neurosurgery, \\ Faculty of Medicine Carl Gustav Carus, Dresden, Germany \\ ${ }^{c}$ Fraunhofer Heinrich-Hertz-Institute, Department of Vision and Imaging Technologies, \\ Berlin, Germany \\ ${ }^{\mathrm{d}}$ Humboldt Universität zu Berlin, Department of Visual Computing, \\ Berlin, Germany
}

\begin{abstract}
Significance: Hyperspectral and multispectral imaging (HMSI) in medical applications provides information about the physiology, morphology, and composition of tissues and organs. The use of these technologies enables the evaluation of biological objects and can potentially be applied as an objective assessment tool for medical professionals.

Aim: Our study investigates HMSI systems for their usability in medical applications.

Approach: Four HMSI systems (one hyperspectral pushbroom camera and three multispectral snapshot cameras) were examined and a spectrometer was used as a reference system, which was initially validated with a standardized color chart. The spectral accuracy of the cameras reproducing chemical properties of different biological objects (porcine blood, physiological porcine tissue, and pathological porcine tissue) was analyzed using the Pearson correlation coefficient.

Results: All the HMSI cameras examined were able to provide the characteristic spectral properties of blood and tissues. A pushbroom camera and two snapshot systems achieve Pearson coefficients of at least 0.97 compared to the ground truth, indicating a very high positive correlation. Only one snapshot camera performs moderately to high positive correlation (0.59 to 0.85 ).

Conclusion: The knowledge of the suitability of HMSI cameras for accurate measurement of chemical properties of biological objects offers a good opportunity for the selection of the optimal imaging tool for specific medical applications, such as organ transplantation.

(C) The Authors. Published by SPIE under a Creative Commons Attribution 4.0 Unported License. Distribution or reproduction of this work in whole or in part requires full attribution of the original publication, including its DOI. [DOI: 10.1117/1.JBO.26.7.076007]
\end{abstract}

Keywords: hyperspectral imaging; multispectral imaging; image-guided surgery; organ analysis; blood analysis; spectral camera comparison.

Paper 210076RR received Mar. 8, 2021; accepted for publication Jun. 24, 2021; published online Jul. 24, 2021.

\section{Introduction}

One of the largest problems for the transplantation medicine is the lack of donor grafts. ${ }^{1}$ Compared to 2010, the rate of postmortem organ donations in Germany has decreased by $29 \%$ in 2019, which corresponds to 1210 organs. Despite an improvement with respect to

\footnotetext{
*Address all coorespondence to Eric L. Wisotzky, eric.wisotzky@hhi.fraunhofer.de

${ }^{\dagger}$ These authors contributed equally to this work.
} 
2017, the need for organs in 2019 (9271 organs required) far exceeds the number of available donor organs (3767 organs transplanted). ${ }^{2}$ This high demand for donor organs shows in particular the need to increase the number of successfully transplanted organs. Optimal use of the few donor organs is urgently required not only with regard to the individual person to be treated but also from a social and political point of view. The most frequently transplanted organs are kidney, liver, heart, and lung. However, in Germany, the discrepancy between supply and demand in kidney transplantation is alarming. A total of 2291 kidneys has been available for transplantation in 2018, compared to 7526 open transplant recipients at the end of the year. ${ }^{3}$ Apart from this shortage, the rejection rate for available kidney transplants has been $14.8 \%$ in $2019 .{ }^{2}$ Due to the lack of an accurate predictive organ evaluation strategy, certain organs were rejected although they would still have been suitable for transplantation. ${ }^{4-7}$ The decision to accept or reject an organ for transplantation depends primarily on the expertise of the transplant team rather than on an objective measurement. In addition in the years 2014 to 2019, intra- or postoperative complications of kidney transplantations requiring a second kidney transplantation have been between $15.3 \%$ and $20.4 \% .^{2,3,8}$ Therefore, an optical evaluation tool to monitor organ quality before transplantation would be of great interest.

The digitization of intraoperative imaging procedures offers the possibility of both the analysis of acquired image data directly during the medical application and the visualization of the automatically extracted information for the surgeon. In this way, information about circulatory disorders, tissue types, or potential structures at risk are obtained during surgery. Hyper- and multispectral imaging (HMSI) are intended to expand the existing intraoperative imaging procedures with the aim of differentiating tissue types as precisely as possible. ${ }^{9,10}$ Spectral cameras record the reflected light components, which can be used to draw conclusions about the physiologically relevant parameters of tissue. Thus HMSI has the potential to measure the optical tissue behavior temporally and spatially resolved. This imaging technique operates among other spectral ranges in the region of the optical window $(\lambda=600$ to $1300 \mathrm{~nm})$, where the main absorbers are melanin, lipids, water, and hemoglobin $(\mathrm{Hb}) .{ }^{9}$ For different biomedical areas, e.g., histology, wound healing, retinal diseases, or cancer detection, the potential of HMSI has been shown. ${ }^{9,11-14}$ It shows the feasibility of detecting variations in $\mathrm{Hb}$ at different oxygen saturation levels ${ }^{14-17}$ of mapping water content in organs ${ }^{18}$ as well as to evaluate organ blood flow characteristics. ${ }^{19,20}$

In organ preservation prior to transplantation, HMSI could be a potential tool to continuously monitor several functional parameters of the organ simultaneously and non-invasively. ${ }^{16,19,21-24}$ Such evaluation of organ quality could help to ensure the appropriate use of donor organs and increase the number of successful transplantations. To evaluate organ quality, different relevant spectral bands have to be analyzed, e.g., water band at $\lambda=970 \mathrm{~nm}$, oxygen band at $\lambda=760 \mathrm{~nm}$, and characteristic Q bands of present porphyrin complexes. ${ }^{25}$ Thus specific markers can be defined to specify organ quality. For example, tissue oxygen saturation $\left(\mathrm{StO}_{2}\right)$ is a promising marker of organ quality. ${ }^{26}$ Further, the optimal blood supply of a transplanted organ is essential for the therapeutic outcome. Currently, the monitoring of such functional parameters and ensuring homogeneous perfusion of a transplanted organ is usually associated with an increased diagnostic effort. This includes contact with the organ and a time-consuming measurement that provides only selective spatial and temporal information. ${ }^{27,28} \mathrm{~A}$ continuous and contactless monitoring of such parameter would make a significant contribution to organ assessment prior and during the transplantation.

For application in different medical fields, especially organ transplantation, HMSI systems have to operate robustly and in a wide spectral range. Therefore, this study compares and evaluates different types of spectral cameras for possible clinical use, such as organ evaluation during transplantation, examining four different porcine organs, and autologous whole blood of three pigs. Thus it can be shown that spectra can be reconstructed using these cameras, which produce useful results for biomedical applications. In the next section, the setup with all cameras as well as its calibration and the scanning process are described. Further, the analyzed organs with its removal and preparation process are presented. The optical properties and the different camera behaviors are presented in Sec. 3. The results of the study are discussed in Sec. 4. 


\section{Materials and Methods}

\subsection{Spectral Camera Specifications}

Four spectral cameras have been used in this study, which are based on two different acquisition techniques. The specifications of each camera and its sensor are presented in Table 1. One camera is a hyperspectral pushbroom camera (Tivita Tissue 8, Diaspective Vision GmbH, Germany), which holds a CMOS sensor and a dispersive element. Each captured two-dimensional image consists of a spatial axis $y$ and the wavelength information $\lambda$. During a scan process, $640 y-\lambda$ images are acquired along the spatial $x$ axis, giving the third dimension of a complete data cube. The system is described in more detail by Mühle et al. ${ }^{14}$

Three cameras are snapshot mosaic cameras using two different sensor setups. Two cameras (MQ022HG-IM-SM4X4-VIS and MQ022HG-IM-SM5X5-NIR, XIMEA GmbH, Germany) hold a $4 \times 4$ - and a $5 \times 5$-mosaic pattern, which includes 16 and 25 wavelength bands, respectively. The $4 \times 4$-mosaic sensor is sensitive in the visible (VIS) spectrum while the $5 \times 5$-mosaic sensor is sensitive in the near-infrared (NIR) spectrum. The $5 \times 5$-mosaic camera contains a longpass filter in front of the optics to cut off all wavelengths $\lambda<675 \mathrm{~nm}$ to avoid secondary bands. Thus each pixel in the mosaic pattern corresponds to one wavelength band. These two cameras are described in more detail by Wisotzky et al. ${ }^{10,29}$ The third snapshot camera (CMS-C, SILIOS, France) holds a $3 \times 3$-mosaic pattern with a slightly different setup. The center pixel of the mosaic pattern is sensitive in the complete sensor sensitivity range and behaves as intensity pixel, whereas the eight surrounding pixels (px) select specific wavelengths $\lambda$ in the VIS spectrum. All three snapshot cameras can handle $60 \mathrm{fps}$ or higher.

In addition to the four camera systems, a spectrometer has been used as a reference system for the analysis of tissue and blood samples. The spectrometer (USB2000 + VIS-NIR-ES, Ocean Optics Inc., USA) with its beam path (QR400-7-VIS-BX, Ocean Optics Inc., USA)

Table 1 The sensor and camera optics specifications of the different spectral camera systems used. The last three parameters have been set during tissue analysis of the porcine organs. The specific bandwidths and spectral resolutions of the three snapshot mosaic cameras can be found in Table S1 in the Supplementary Material.

\begin{tabular}{lcccc}
\hline \hline Camera & Pushbroom & Snapshot $4 \times 4$ & Snapshot $5 \times 5$ & Snapshot $3 \times 3$ \\
\hline Sensor & CMOS & CMOS & CMOS & CMOS \\
Sensor ADC (bits) & 12 & 10 & 10 & 10 \\
Sensor resolution (px) & $1280 \times 960$ & $2048 \times 1088$ & $2048 \times 1088$ & $1280 \times 1024$ \\
Output resolution (px) & $640 \times 480$ & $2048 \times 1024$ & $2045 \times 1080$ & $1280 \times 1024$ \\
Sensor size (mm) & $4.8 \times 3.6$ & $11.3 \times 6.0$ & $11.3 \times 6.0$ & $6.8 \times 5.5$ \\
Pixel size ( $\mu$ m) & 3.75 & 5.5 & 5.5 & 5.3 \\
Focal length & $12 \mathrm{~mm} \mathrm{f} / 1.4$ & $75 \mathrm{~mm} \mathrm{f} / 2.8$ & $75 \mathrm{~mm} \mathrm{f} / 2.8$ & $35 \mathrm{~mm} \mathrm{f} / 1.65$ \\
Spectral range (nm) & 500 to 995 & 463 to 638 & 693 to 966 & 430 to 700 \\
No. of bands & 100 & $16(4 \times 4)$ & $25(5 \times 5)$ & $8+1(3 \times 3)$ \\
Bandwidth (FWHM avg.) (nm) & 5 & 15 & 15 & 40 \\
Spectral resolution (nm) & 5 & 8 to 12 & 8 to 12 & 27 to 41 \\
Frame rate (fps) & 60 & 20 & 20 & 12 \\
Exposure time (ms) & 0.25 & 50.0 & 50.0 & 83.3 \\
WD (mm) & 403 & 319 & 319 & 272 \\
\hline \hline
\end{tabular}


is an asymmetrical Czerny-Turner monochromator using a CCD detector. It has a spectral resolution of $1 / 3 \mathrm{~nm}$ and covers a wavelength range of 350 to $1000 \mathrm{~nm}$.

\subsection{Imaging Setup}

The acquisition setup with all four cameras and illumination spots is shown in Fig. 1. The illumination unit contains six 20-W quartz-tungsten-halogen spots (OSRAM Decostar 51 ALU, Osram GmbH, Germany) with an aluminum reflector and a defined beam angle and beam position (Fig. 1, No. 5). Each spot has a luminosity of $510 \mathrm{~cd}$ and a color temperature of $2800 \mathrm{~K}$. All spots are mounted in a distance of $450 \mathrm{~mm}$ to the target and combined having an illuminance of 12.2 klux in that working distance (WD).

All cameras (Fig. 1, Nos. 1 to 4) focus on the same target area, which is indicated by a cursor defining a circular region of interest (ROI) of $d=30 \mathrm{~mm}$ (Fig. 1, No. 10). Snapshot cameras acquire the entire wavelength information in one shot resulting in a spatial/spectral $2 \mathrm{D}$ representation of the dataset but with a reduced spatial resolution and fewer spectral bands ( 8 to 25 for the used cameras in this study). As the two Ximea snapshot cameras (Fig. 1, Nos. 2 and 3) are mounted on one static plate (stereo-like) and cover different spectral intervals, i.e., $\lambda=463$ to $638 \mathrm{~nm}$ for the $4 \times 4$-VIS and $\lambda=693$ to $966 \mathrm{~nm}$ for the $5 \times 5$-NIR camera, respectively, the data of both cameras are combined to one spectral dataset using pixel correspondences between the two cameras calculating a disparity map ${ }^{30-33}$ (hereinafter referred to as multispectral 41-bands setup) and are then analyzed. The Silios snapshot camera (Fig. 1, No. 4) covers the same spectral interval as the $4 \times 4$-VIS camera and furthermore covers the gap between the two Ximea cameras. Hence, it is used as a comparable $3 \times 3$-VIS setup. This allows an estimation about the minimum required number of spectral bands (16 bands of $4 \times 4$-VIS versus 8 bands of $3 \times 3$-VIS) for robust organ surveillance. The pushbroom camera (Fig. 1, No. 1) captures the complete spectral dataset of a recorded slit in one shot. The second spatial component is created by moving the slit. The recorded data cube consists of 640 pixels in the traverse direction of the slit and 480 pixels in the direction of the slit. The angle between the illumination units and the different cameras is in the range of $\alpha \approx 25 \mathrm{deg}$ to $35 \mathrm{deg}$. For reference data, a spectrometer (USB2000 + VIS-NIR-ES, Ocean Insight, USA) measures the object in the circular area (Fig. 1, No. 6). The spectrometer points toward a small region at the border of the circular area. As the spectrometer has no local resolution, the reference spectrum is averaged over the entire measurement area.
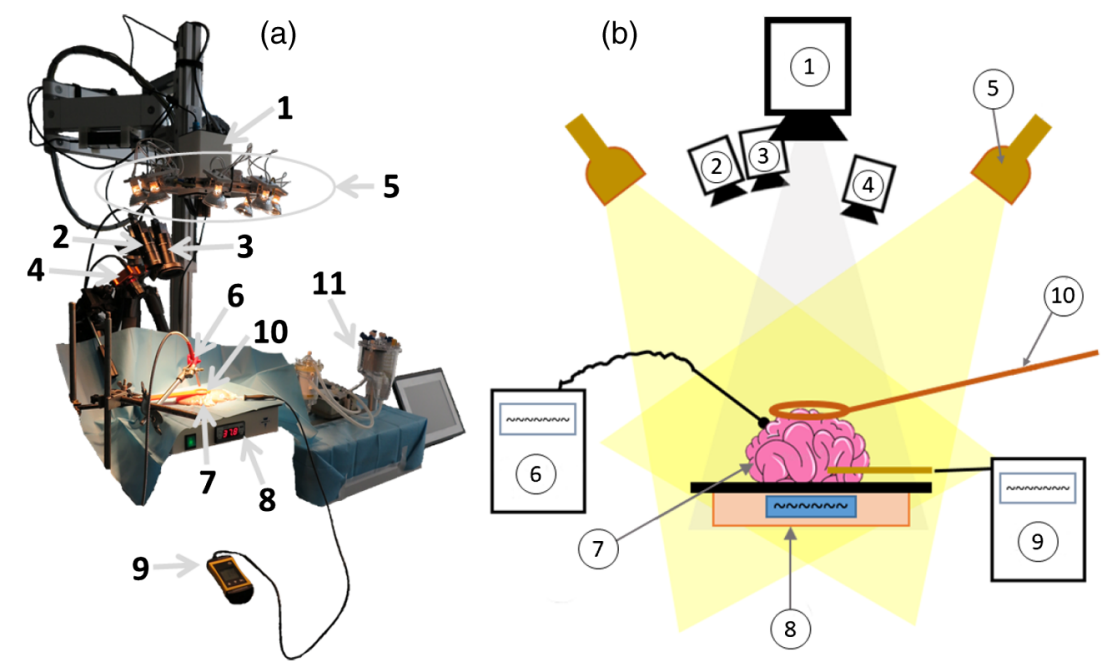

Fig. 1 The setup is shown during (a) calibration and (b) as sketch with the complete equipment: (1) pushbroom camera setup, 41-bands setup with (2) $4 \times 4$-VIS camera and (3) $5 \times 5$-NIR camera, (4) $3 \times 3$-VIS camera setup, (5) six illumination spots, (6) spectrometer, (7) tissue sample, (8) heating unit, (9) thermometer, (10) cursor, and (11) perfusion machine for blood measurements. 
To obtain the reflectance spectrum from the measured raw data, each image has to be corrected according to

$$
I_{\text {reflectance }}=\frac{I_{\text {raw }}-I_{\text {dark }}}{I_{\text {white }}-I_{\text {dark }}},
$$

where $I_{\text {reflectance }}$ is the resulting reflectance spectrum, $I_{\text {raw }}$ is the measured raw data, $I_{\text {dark }}$ contains the dark reference data, and $I_{\text {white }}$ is the white reference intensity spectrum, as described in particular by Mühle et al. ${ }^{14}$ and Wisotzky et al. ${ }^{10,29}$

\subsection{Organ and Blood Analysis}

We have analyzed tissues of typical donor organs (heart, lung, and kidney) to compare the four introduced spectral cameras. In addition, brain and blood have been analyzed as well. All organs used in this study have been considered healthy by the veterinarian present at the time of collection. In addition, one kidney affected with cysts is collected. All organs are retrieved from three female German Landrace pigs weighting 100 to $120 \mathrm{~kg}$. The cyst-affected kidney is retrieved from the first pig, which is the only pig where both kidneys, a healthy and a cystaffected kidney, have been collected. After death by exsanguination, all organs and the blood have been collected. Approximately 1.51 of blood has been received from the carotid incision into a receptacle containing 10,000 units of heparin (Rotexmedica GmbH, Trittau, Germany). The blood has been stored at $4^{\circ} \mathrm{C}$. All other organs have been removed en bloc and, with exception of the brain, flushed immediately with preservation solution (Custodiol, Dr. Franz Köhler Chemie GmbH, Bensheim, Germany). Afterward, the organs have been placed into a bag with preservation solution and stored on ice at $4^{\circ} \mathrm{C}$. For blood collection, the time between exsanguination and storing on ice has been $\sim 2$ min. The process of organs and blood removal is described in more detail by Markgraf et al. ${ }^{23}$ The warm ischemia time occuring between interruption of blood supply and storage on ice has been $\sim 44.5 \pm 0.5 \mathrm{~min}$ for each porcine model. Before starting the measurement procedure, the organs have been removed from ice after $\sim 1$ up to $3.5 \mathrm{~h}$ cold ischemia time (CIT) and are heated up to the target temperature of $\sim 37^{\circ} \mathrm{C}$. The heating process has been carried out for $25 \mathrm{~min}$ in a water bath. The procedure described in this paragraph has been adapted to simulate a state-of-the-art clinical transplantation procedure, where the spectral measurement would be performed 25 min after organ reperfusion.

\subsection{Measurement Procedure}

At the beginning, reference measurements with a color chart (ColorChecker Classic, X-Rite Inc.) have been performed to set up the optimal camera and spectrometer settings, such as exposure time, focus, or frame rate, for each device (cf., Table 1). In addition, these measurements have been used to validate the spectrometer measurements as the colored tiles hold well-defined color spectra. ${ }^{34}$ For each colored tile, the reflectance spectrum is known in the range of 390 to $1000 \mathrm{~nm}$ with step size of $10 \mathrm{~nm} .{ }^{35}$ Because of this validation, we can use the spectrometer measurements as ground truth or reference data for the organ measurements. The spectrometer measurements are performed by averaging 10 scans, a Boxcar width of 2 , an integration time of 8 ms per scan, and a distance of $3.5 \mathrm{~cm}$ with angle of $12.5 \mathrm{deg}$ to the target area having diameter of $1.95 \mathrm{~cm}$. The software OceanView 1.6.3 (Lite, Ocean Optics Inc., USA) has been used to record and process the raw data.

Then all organs of each porcine model are measured successively in the order brain, kidney, lung, and heart. After each heating process, the organs are placed under the cursor (Fig. 1, No. 10) on a heating unit (Fig. 1, No. 8) to ensure a constant temperature during the measuring process. A thermal sensor (Fig. 1, No. 9) is placed inside the organs to control the core temperature during the measurements. For calibration purposes [cf., Eq. (1)], white and dark images are acquired before as well as after each organ measurement to achieve $I_{\text {white }}$ and $I_{\text {dark }}$, respectively. For acquisition of $I_{\text {white }}$, a white standard (99\% reflectance Zenith, SphereOptics GmbH, Germany) is recorded simultaneously with all cameras and the spectrometer using the same 
illumination. Dark images are acquired after occluding the optics of all cameras and the spectrometer.

For each organ as well as the blood samples, three measurements have been performed with all cameras simultaneously. Each camera captures the ROI bordered by the cursor (Fig. 1, No. 10; cf., Fig. 4). One pushbroom measurement takes about $10 \mathrm{~s}$. In parallel, 10 images have been acquired for each of the snapshot cameras. These 10 images are averaged to one dataset. Four different healthy organs of three pigs, one additional kidney affected with cysts as well as blood with three different oxygenation conditions have been scanned. Consequently, a total of 48 measurements has been performed for each camera: 36 healthy organ measurements, 3 cyst-affected kidney measurements, and 9 blood measurements. The blood conditioning experiments have been carried out in accordance to Markgraf et al. ${ }^{23}$ and three different oxygenation saturation levels $(98.6 \%, 92.8 \%$, and $82.0 \%)$ have been set up. Since specular reflections are unavoidable in the ROI marked by the cursor, for the analysis, two smaller areas in the ROI are selected manually by biomedical experts considering the anatomical structures in each measured image of each camera, which contain no interfering artifacts. These two areas represent the similar location over all cameras per measurement. Each area has a minimum size of $21 \mathrm{px} \times 21 \mathrm{px} \times N$ bands, where $N$ is the number of possible wavelength bands dependent on the used camera. The measured sensor data of these ROIs are averaged for further evaluation in MATLAB R2019b (The MathWorks Inc., Natick, Massachusetts, USA).

\section{Results}

\subsection{Color Checker Measurements}

Reference measurements with the color chart are applied to validate the spectrometer measurements using published reflectance data of the colored tiles. Several characteristic color tiles, showing different spectral distributions, have been scanned. Five representative reflectance data are shown in Fig. 2. The comparison between the spectra of the spectrometer and the known spectra of the color chart shows comparable results. The highest deviation between these spectra is $5 \%$. In addition, two further color tiles are measured with the three camera setups to evaluate the comparability of the achieved reflectance data on a standardized, non-biological test object. The data recorded simultaneously by the four spectral cameras as well as the spectrometer have slightly different formats. Four spectral curves are achieved from the measurements (cf., Table 1): (1) spectrometer reference data $(\lambda=400$ to $1000 \mathrm{~nm}$ with step size $1 / 3 \mathrm{~nm})$, (2) hyperspectral pushbroom camera ( $\lambda=500$ to $995 \mathrm{~nm}$ giving 100 bands in step size $5 \mathrm{~nm})$, (3) multispectral 41-bands setup ( $\lambda=463$ to $966 \mathrm{~nm}$ giving 41 bands), and (4) multispectral $3 \times 3$-VIS snapshot camera ( $\lambda=430$ to $700 \mathrm{~nm}$ giving eight bands). The camera setup results of the two

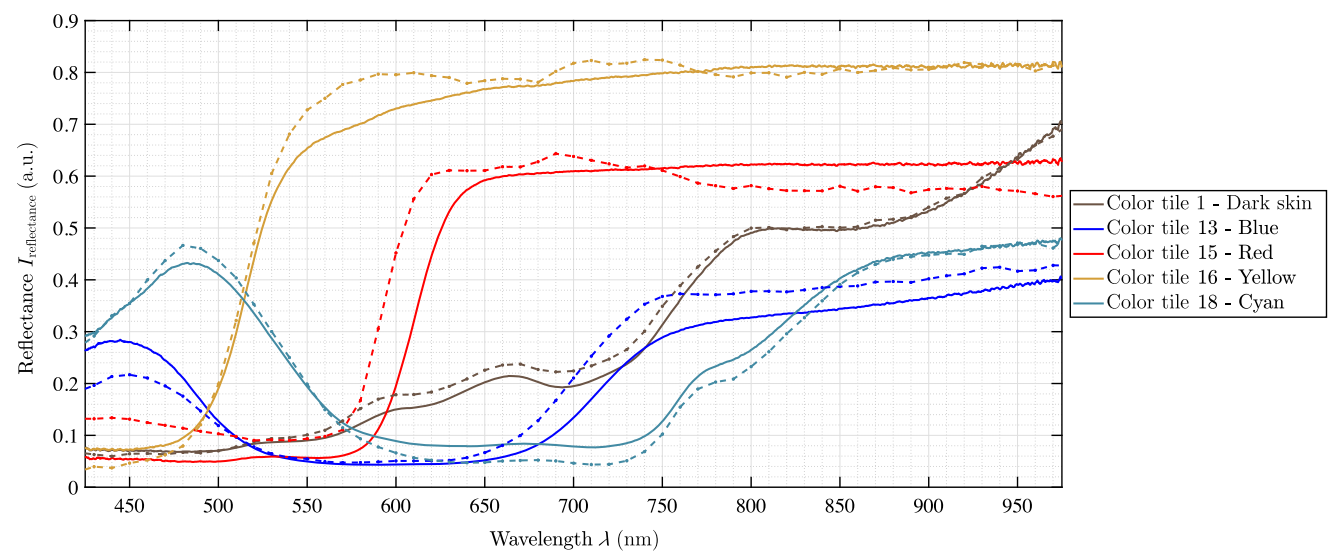

Fig. 2 Five different analyzed color tiles of the Color Checker Board. The continuous lines are measured with a spectometer in this study and the dashed lines are published reference measurements. ${ }^{34,35}$ 


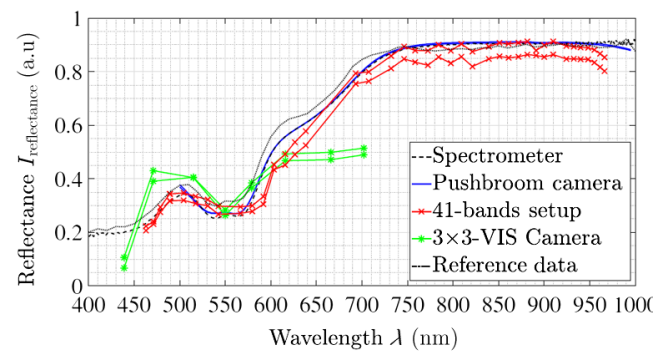

(a)

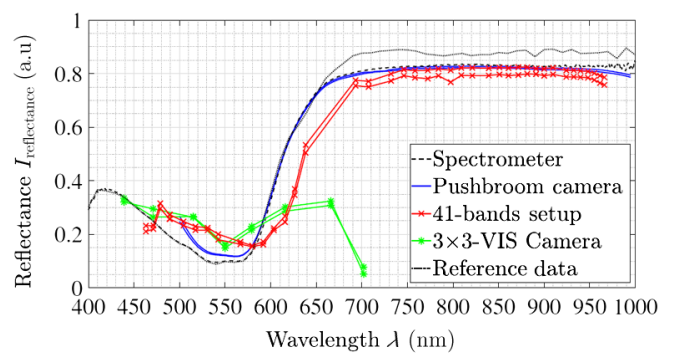

(b)

Fig. 3 Two color tiles, with defined colors (a) light skin and (b) magenta, analyzed with all four described setups. The two curves per imaging setup correspond to the two selected ROls in each captured image.

color tiles are shown in Fig. 3. The pushbroom camera data as well as the 41-bands setup data follow the published color spectra as well as the ground truth spectrometer data. The multispectral $3 \times 3$ camera data show more variability in the eight reconstructed data bands. In the following, these behaviors are presented and discussed in detail based on the organ measurements of the three analyzed pigs.

\subsection{Organ Measurements}

The measurements of the different porcine organs have been performed in a time range of approx. $t=2 \mathrm{~h}$, i.e., $t=2 \mathrm{~h}$ to $t=4 \mathrm{~h}$ after collection. Thus the CIT has been in the range of $t_{\mathrm{CIT}}=63 \mathrm{~min}$ and $t_{\mathrm{CIT}}=214 \mathrm{~min}$. The core temperatures $T_{\text {core }}$ of the healthy organs during the measurements are presented in Table 2. Organ core temperatures $T_{\text {core }}$ have ranged between $T_{\text {core }}=31.3^{\circ} \mathrm{C}$ and $T_{\text {core }}=36.1^{\circ} \mathrm{C}$.

A visualization of the captured data for the heart of the third animal is shown in Fig. 4. With the hyperspectral pushbroom camera, the largest field-of-view could be captured, followed by the multispectral $3 \times 3$-VIS camera and finally by the multispectral $4 \times 4$ VIS and $5 \times 5$-NIR cameras. With all four camera systems, the measuring object field given by the cursor can be successfully recorded.

For each of the four porcine organs, a similar spectral behavior is captured through all four different acquisition methods in the corresponding spectral intervals of $\sim 450$ up to 700 or $1000 \mathrm{~nm}$, respectively (see Fig. 5). All organs show a local peak at about $\lambda \approx 485 \mathrm{~nm}$, a local minimum at about $\lambda \approx 555 \mathrm{~nm}$, and a rising edge in the range of $\lambda \approx 600 \mathrm{~nm}$ to $\lambda \approx 630 \mathrm{~nm}$. The maximal reflectance intensity of the spectra is at $\lambda \approx 800 \mathrm{~nm}$. In addition, the different organs have specific spectral variations (see Fig. 5). The reflectance data averaged over all measurements results in an $\sim 50 \%$ lower reflectance intensity for the heart over the entire spectrum compared to the other three spectra in an overall constant setting for all organs. Further, the reflectance variation over the entire analyzed spectrum is smaller for the porcine kidney and porcine heart samples compared to the lung and brain samples. Brain and lung data are comparable with specific differences. The brain spectra shows an intensity decrease in the infrared

Table 2 Organ core temperature $T_{\text {core }}\left({ }^{\circ} \mathrm{C}\right)$ recorded during the measurements of kidney, lung, heart, and brain in the three porcine models as well as blood temperature $\left({ }^{\circ} \mathrm{C}\right)$ of different blood oxygen saturation levels (\%). The different oxygenation levels of the blood sample are achieved via a perfusion oxygenator setup. ${ }^{23}$

\begin{tabular}{lcccccc}
\hline \hline Pig & Kidney $\left({ }^{\circ} \mathrm{C}\right)$ & Lung $\left({ }^{\circ} \mathrm{C}\right)$ & Heart $\left({ }^{\circ} \mathrm{C}\right)$ & Brain $\left({ }^{\circ} \mathrm{C}\right)$ & $\mathrm{sO}_{2}(\%)$ & Blood $\left({ }^{\circ} \mathrm{C}\right)$ \\
\hline 1 & 31.3 & 32.5 & 35.0 & 35.7 & 82.0 & 37.1 \\
2 & 33.8 & 33.3 & 35.7 & 36.1 & 92.8 & 36.9 \\
3 & 35.1 & 32.8 & 34.5 & 33.1 & 98.6 & 37.3 \\
\hline \hline
\end{tabular}




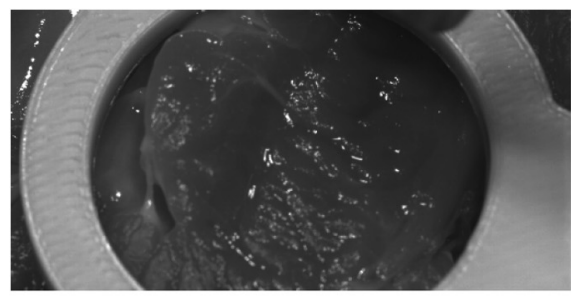

(a)

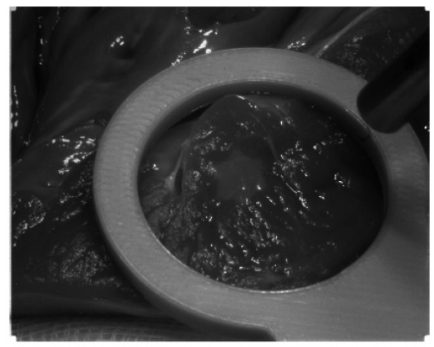

(c)

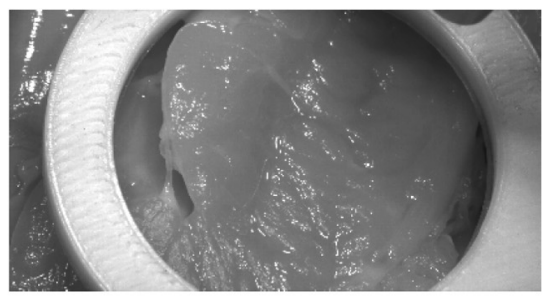

(b)

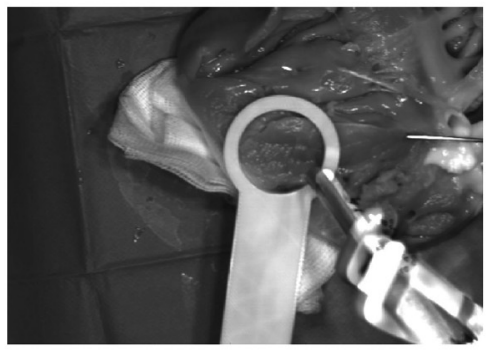

(d)

Fig. 4 Spatially resolved HMSI images of the heart of the third pig model. (a) Multispectral $4 \times 4$ VIS camera; (b) multispectral $5 \times 5$ NIR camera; and (c) Multispectral $3 \times 3$ VIS camera. One spectral band of the snapshot sensor data for (a) $\lambda=530 \mathrm{~nm}$, (b) $\lambda=746 \mathrm{~nm}$, and (c) $\lambda=515 \mathrm{~nm}$. (d) The intensity distribution of the TIVITA Tissue pushbroom camera image at $\lambda=750 \mathrm{~nm}$.

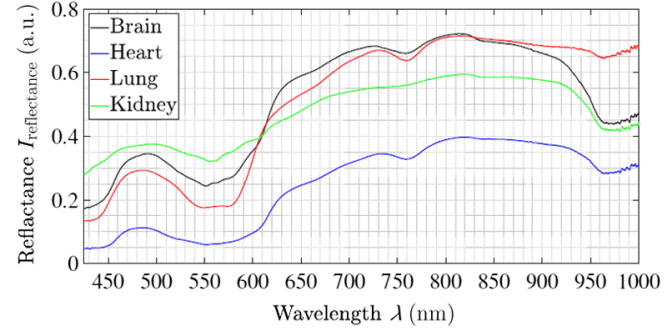

(a)

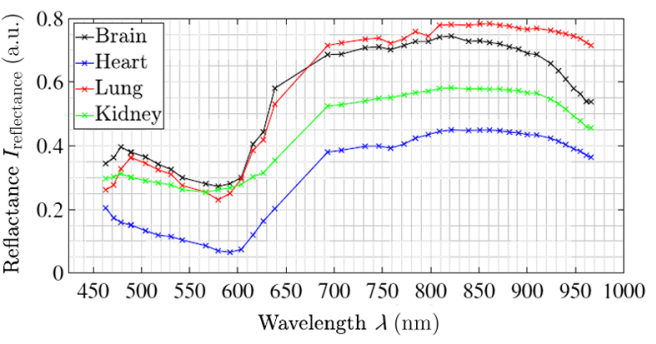

(c)

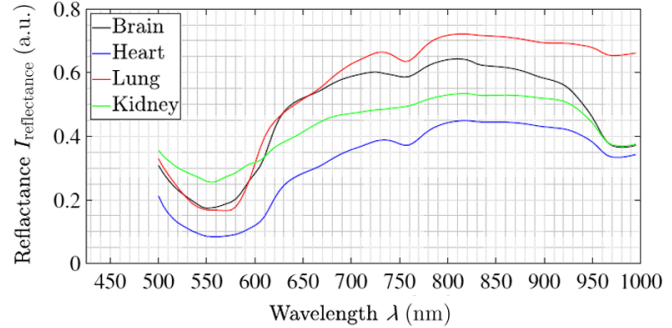

(b)

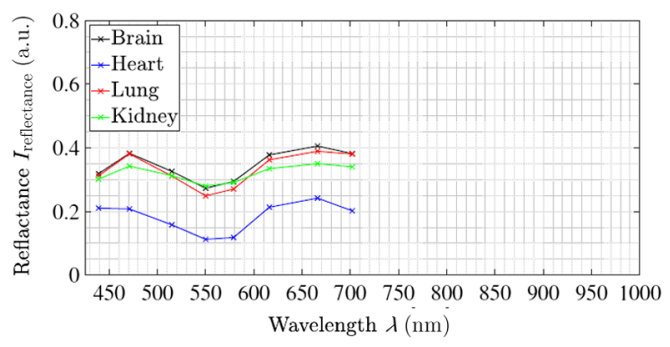

(d)

Fig. 5 The spectral reflectance behaviors for all four analyzed organs averaged over all measurements presented of (a) spectrometer ground truth data, (b) hyperspectral pushbroom camera, (c) multispectral 41-band setup, and (d) multispectral $3 \times 3$ camera.

(IR) range at $\lambda=950 \mathrm{~nm}$, whereas it has slightly higher intensity in the visible range $(\lambda=460 \mathrm{~nm}$ to $\lambda=600 \mathrm{~nm}$ ) in comparison to lung data. The reflection spectra of the kidney are comparable to the brain and lung spectra and intersect them at $\lambda=600 \mathrm{~nm}$. It shows a flat trend in the NIR and IR range, with a decrease at $\lambda=950 \mathrm{~nm}$ similar to the heart spectrum.

In detail, differences through the spectral behavior of the organs exist over the complete analyzed spectra. While heart, kidney, and brain show a different decrease of intensity starting at $\lambda=950 \mathrm{~nm}$, the spectrum of the lung remains constant. Thus the lung shows a strongly increased reflectance from about $\lambda=950 \mathrm{~nm}$ compared to the other three organs. 
Furthermore, the rising band at $\lambda \approx 600$ to $630 \mathrm{~nm}$ is steep for heart, lung, and brain, whereas the increase in reflectance before and after that band is smooth. In addition, these organs show a differently pronounced local peak at $\lambda \approx 730 \mathrm{~nm}$ and a local minimum at $\lambda \approx 757 \mathrm{~nm}$. Considering the kidney dataset, the spectrum results are smoother, with a monotonic increase in reflectance from $\lambda \approx 560 \mathrm{~nm}$ up to $\lambda \approx 685 \mathrm{~nm}$. After this point, the reflectance remains

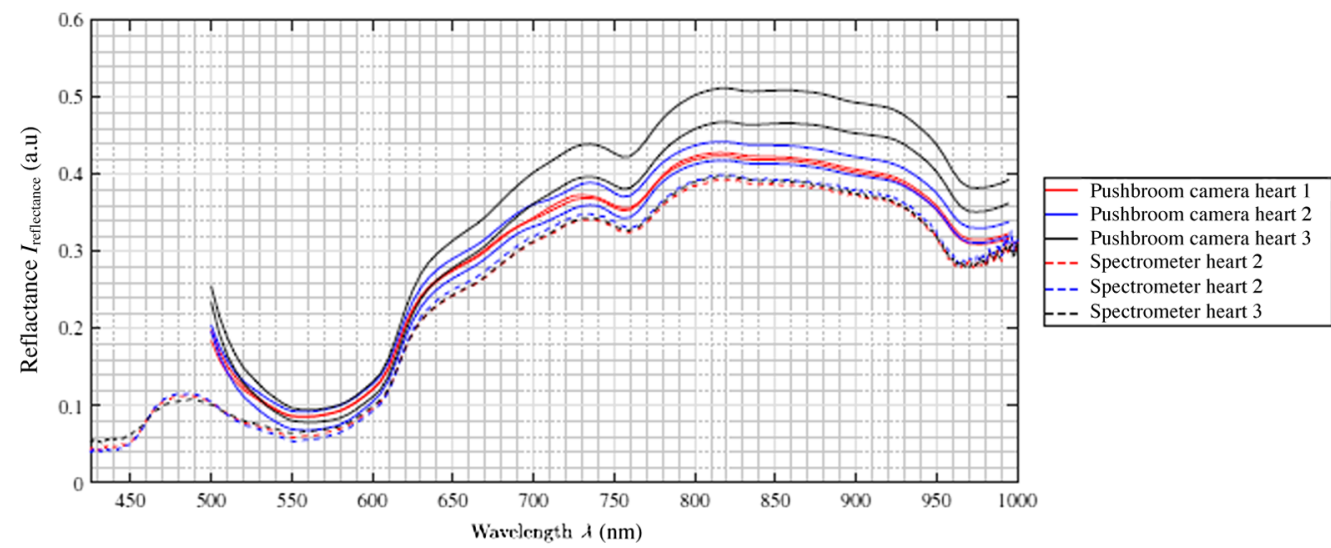

(a)
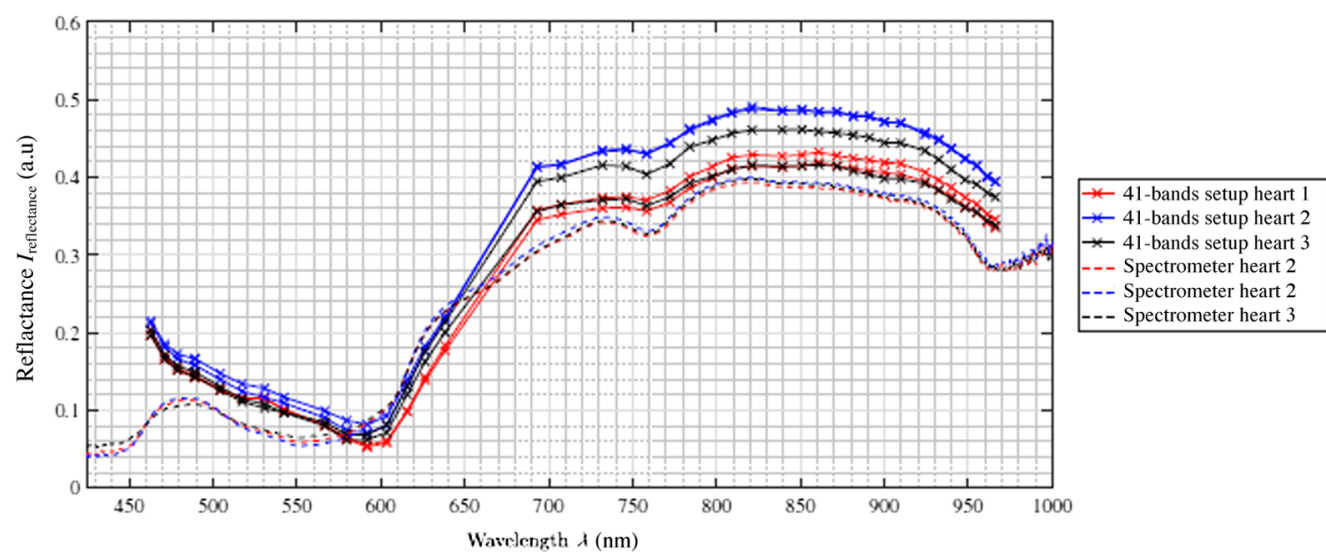

(b)

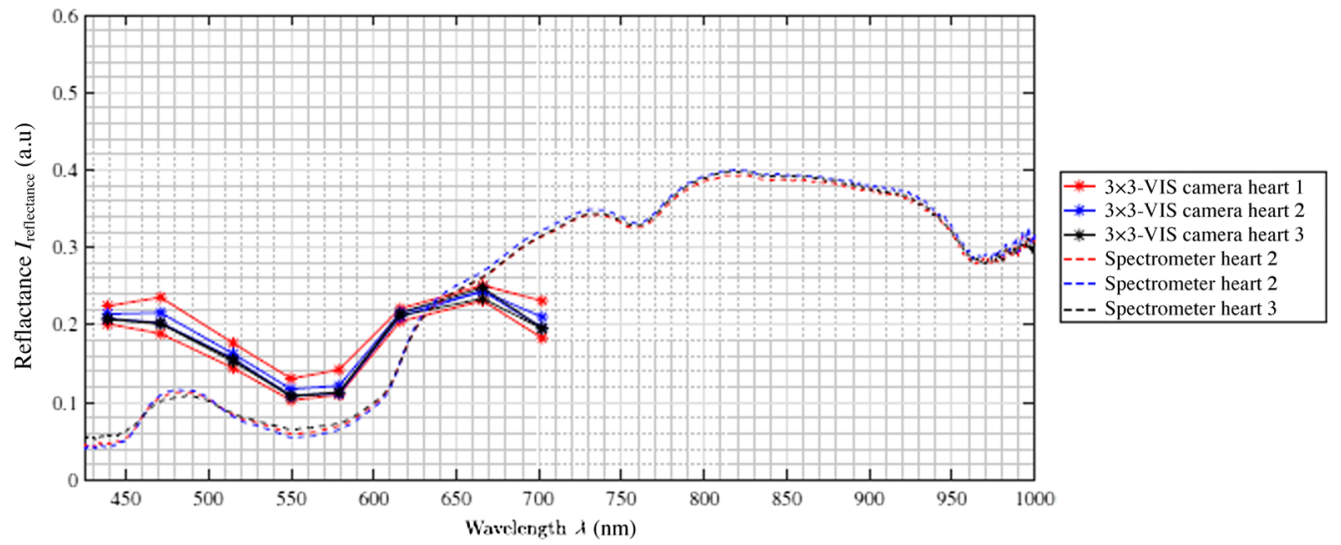

(c)

Fig. 6 For the porcine heart measurements, all curves acquired by the different spectral cameras-(a) hyperspectral pushbroom camera, (b) 41-bands setup, and (c) $3 \times 3$-VIS cameramatch the behavior of the reference spectrometer data (dashed lines). For each of the three camera acquisition methods, two ROIs are selected, reconstructed, and averaged over the three measurements. The resulting two ROIs are presented here. 
relatively flat showing no pronounced local maxima or minima, different from the other organ samples.

All the obtained spectra are consistent with the spectral properties of common tissue chromophores in the range of 400 to $1000 \mathrm{~nm}$. The primary light-absorbing compounds in tissue within this range are $\mathrm{Hb}$ and water. Depending on different oxygen conditions, a conformational change occurs in the $\mathrm{Hb}$ molecule, which leads to spectral changes in the UV-VIS-NIR range. The spectrometer data display in the range of $\lambda=380$ to $500 \mathrm{~nm}$ the extreme intense Soret bands. ${ }^{36}$ The minima at $\lambda=555 \mathrm{~nm}$ and $\lambda=765 \mathrm{~nm}$ in the reflectance curve are characteristic bands of the porphyrin $\mathrm{Hb}$ molecule. ${ }^{36}$ The flattening of the spectral curve from $\lambda=600 \mathrm{~nm}$ to $\lambda=740 \mathrm{~nm}$ as well as the formation of two minima in the range from $\lambda=500 \mathrm{~nm}$ to $\lambda=600 \mathrm{~nm}$ are spectral features of $\mathrm{HbO}_{2}$. For the lung data, the double peak of $\mathrm{HbO}_{2}$ is visible in the spectrometer, pushbroom, and 41-band data. The $3 \times 3$-VIS data contain too few $\lambda$-bands to resolve the characteristic spectral properties. The observed local minima at $\lambda=760 \mathrm{~nm}$ and $\lambda=970 \mathrm{~nm}$ correspond to water-specific spectral properties, e.g., the absorption peaks of water. ${ }^{25}$

Both the individual organ spectra of the camera measurements and the spectrometer measurements vary in relation to the reflectance intensity (i.e., intensity axis in Figs. 5-9). This behavior can also be detected in the spectrometer reference measurement of the three pigs. However, the measured and reconstructed 41-bands setup reflectance [cf., Fig. 5(c)] and the reflectance of the pushbroom camera [cf., Fig. 5(b)] lie within these reference ranges and correspond to the optical behavior of the organ. To quantify the similarity between the spectra of the spectral cameras and the spectra of the reference a normalized cross correlation (NCC),

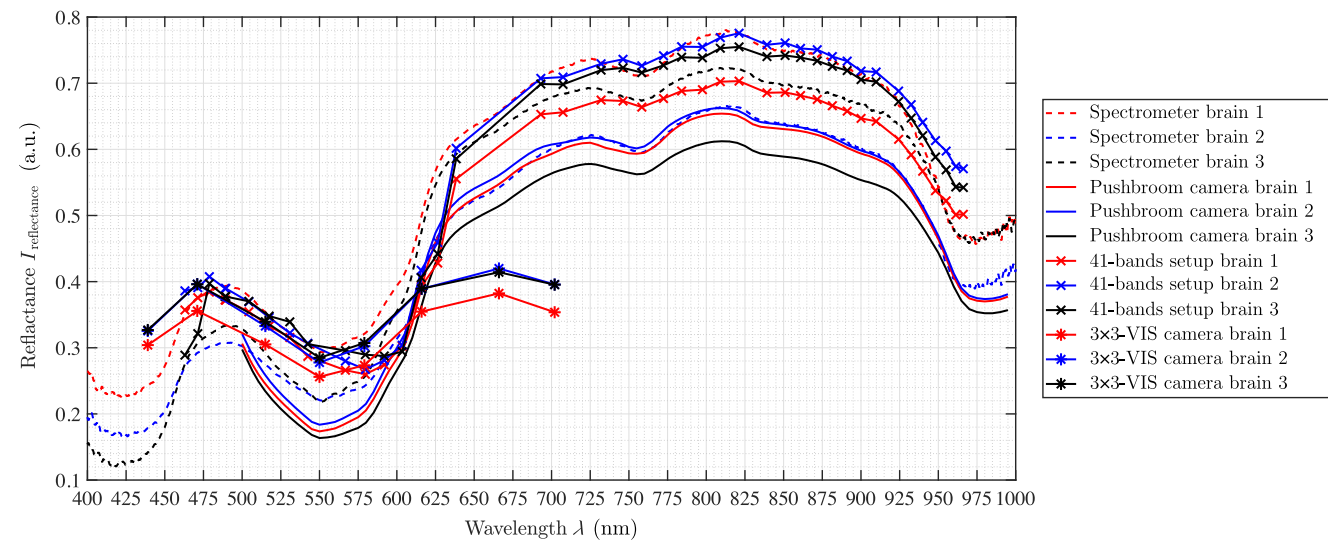

Fig. 7 Porcine brain measurements of all three pigs both two selected ROls are averaged to one spectral curve.

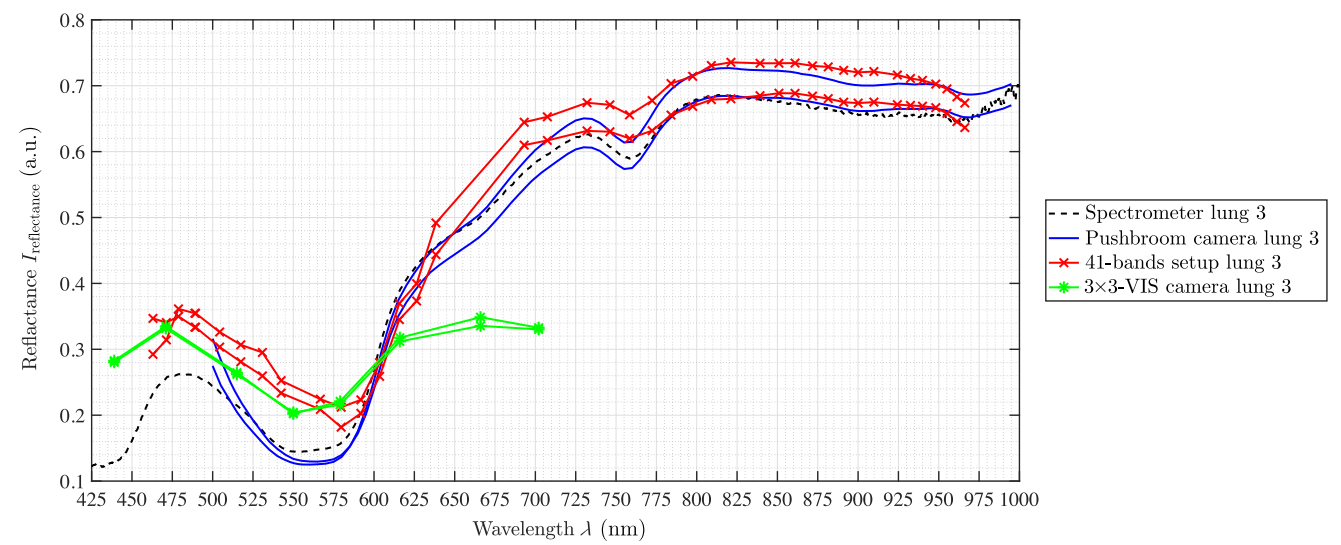

Fig. 8 Porcine lung measurements of the third pig at two different selected ROls. These spectral curves are representative for the other two pigs as well. 


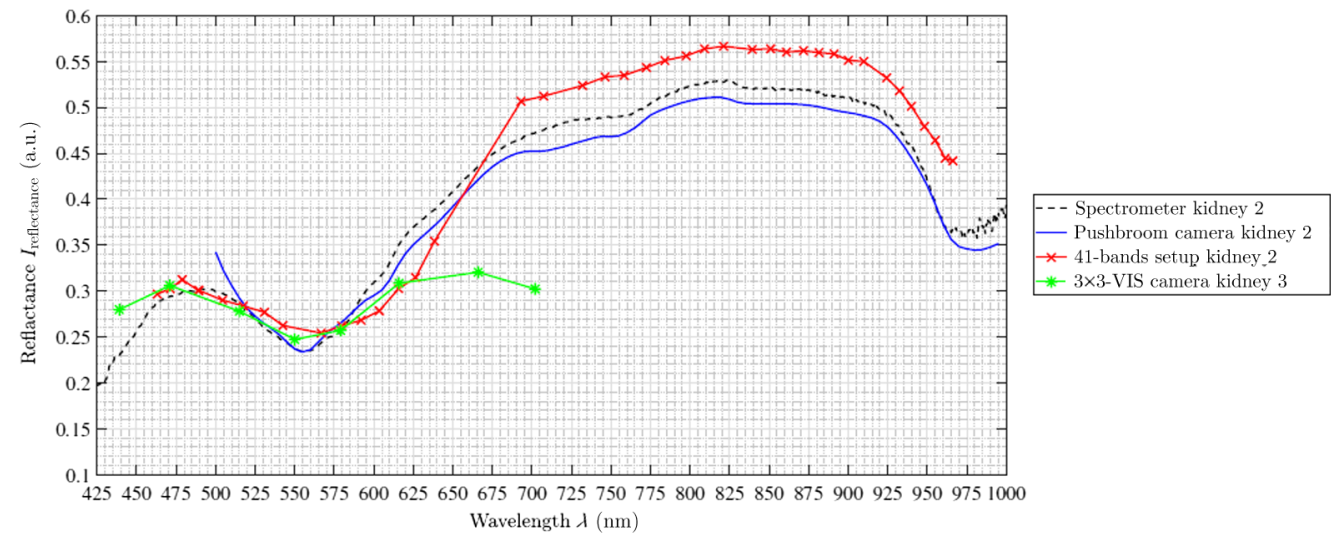

(a)

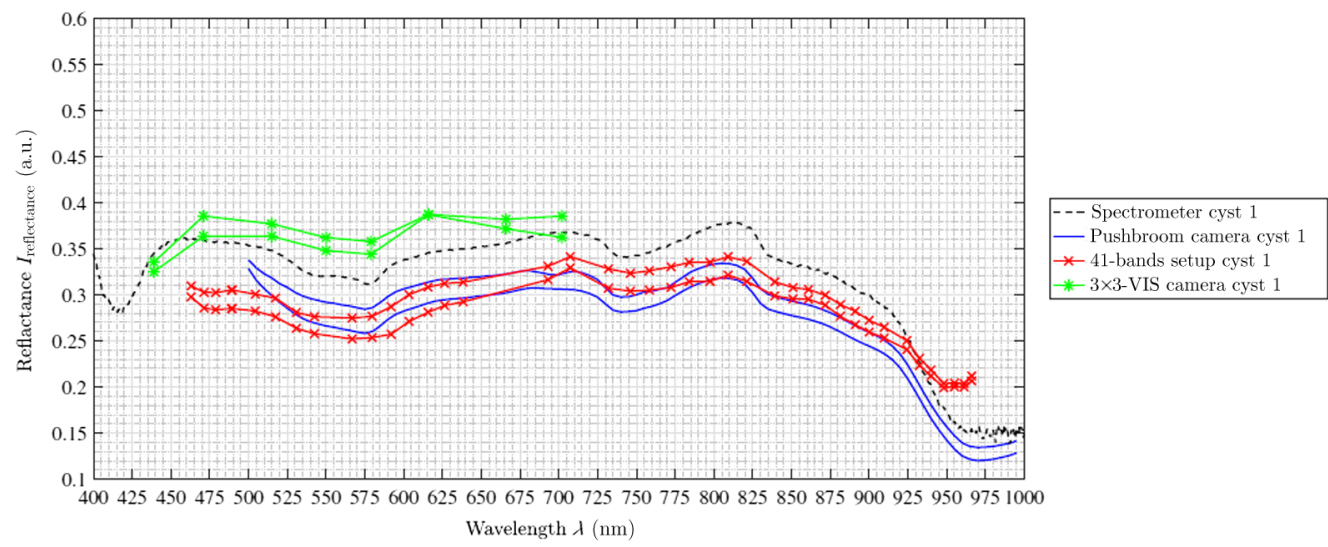

(b)

Fig. 9 Porcine kidney measurements of (a) healthy kidney tissue from the second pig and (b) kidney with a superficial cyst from the first pig, both averaged over both measured ROls. The spectral curves in (a) are representative for the other two pigs as well, which are omitted for readability. In (b), the spectrometer data of the healthy kidney of the first pig are plotted for comparability.

i.e., Pearson correlation coefficient, is applied (see Table 3). All NCC metrics are in the range of 0.97 up to 0.99 for the pushbroom camera and 41-bands setup data. The difference of about $1.5 \%$ for NCC values between the comparisons of spectrometer and pushbroom or 41-bands setup (0.995 versus 0.980 ) comes along the linear fitting between the single bands with wider band distances for the 41-bands setup data. In addition, the comparisons between the single camera

Table 3 NCC between the averaged spectral curves of the different acquisition methods. The curves of the pushbroom, the 41 -bands, and the $3 \times 3$-VIS camera setup are linear fitted between the single bands. The number of data points indicates the used points for NCC calculation.

\begin{tabular}{|c|c|c|c|c|c|c|}
\hline Camera comparison & Kidney & Lung & Heart & Brain & $\lambda$-area $(\mathrm{nm})$ & Points \\
\hline Spectrometer versus pushbroom & 0.9938 & 0.9978 & 0.9957 & 0.9982 & 500 to 995 & 1518 \\
\hline Spectrometer versus 41 -bands & 0.9707 & 0.9822 & 0.9817 & 0.9820 & 460 to 970 & 1540 \\
\hline Spectrometer versus $3 \times 3$-VIS & 0.8369 & 0.8397 & 0.5894 & 0.8533 & 400 to 700 & 850 \\
\hline Pushbroom versus 41 -bands & 0.9782 & 0.9841 & 0.9870 & 0.9824 & 500 to 970 & 1434 \\
\hline Pushbroom versus $3 \times 3$-VIS & 0.9408 & 0.9718 & 0.8975 & 0.9566 & 500 to 700 & 579 \\
\hline 41 -bands versus $3 \times 3$-VIS & 0.7378 & 0.7621 & 0.7376 & 0.8250 & 460 to 700 & 685 \\
\hline
\end{tabular}


setups are performed to characterize the comparability between the three different acquisition setups. The Pearson coefficients between pushbroom and 41-bands setup are in the range of $0.985 \pm 0.005$, which shows a robust comparability between these two setups.

The measured and reconstructed reflectances of the $3 \times 3$-VIS camera [cf., Fig. 5(d)] are different to the other two camera acquisition methods. The captured spectral data are located exclusively in the VIS wavelength range between $\lambda=425 \mathrm{~nm}$ to $700 \mathrm{~nm}$. The first six wavelength bands in the range of $\lambda=425$ to $625 \mathrm{~nm}$ correspond to both the spectrometer reference data and the other two camera systems. Only for the heart measurements (cf., Fig. 6), the reflectance intensity of the first band is too high and do not fit the reference. Furthermore, the last two measured and reconstructed wavelength bands at approx. $\lambda=665 \mathrm{~nm}$ and $\lambda=700 \mathrm{~nm}$ show consistently too low reflectance intensities for all organs (cf., Figs. 5-9).

The NCC results of the $3 \times 3$-VIS camera data substantiate these appearances (see Table 3 ). When comparing $3 \times 3$-VIS camera with the reference spectrometer, the Pearson coefficient reaches $0.84 \pm 0.01$ for kidney, lung, and brain measurements and 0.59 for heart measurements. Due to the data fitting for the pushbroom setup as well as for the $3 \times 3$-VIS camera setup, the NCC is higher when comparing these setups ( 0.90 up to 0.97). Compared to the multispectral 41-bands setup, the NCC data are reduced, in a range of 0.74 to 0.83 , as for both acquisition methods less data points are used for fitting.

The spectra of the porcine hearts are similar for all different recordings methods, i.e., spectrometer and different setups, and through all different measurements, shown in Table 3 and Fig. 6 . The first data points of the spectra of the hyperspectral pushbroom camera $(\lambda \approx 500$ to $515 \mathrm{~nm}$ ) as well as the first two spectral bands of the $4 \times 4$-VIS camera of the 41-bands setup show a strong scattering or inaccurate behavior. This is visible for all measurements across all organs (cf., Figs. 7 and 8) for brain of all three pigs and lung of the third pig, respectively. Further, the 41-bands dataset consists of a large gap in the spectral range of $\lambda=640 \mathrm{~nm}$ and $\lambda=690 \mathrm{~nm}$. Therefore, in this region, the spectral behavior cannot be represented correctly, as this gap is the transition between the two combined multispectral cameras.

Especially, the multispectral snapshot cameras show some discontinuities in the reconstructed reflectance bands, as already described for the first two spectral bands of the $4 \times 4$ VIS of the 41-bands setup. Further, the band at $\lambda=630 \mathrm{~nm}$ always underestimates the reflectance intensity. In contrast, all bands of the $5 \times 5$-NIR represent the spectral organs behaviors with all local maxima and minima precisely.

In addition to the healthy porcine organs, one kidney affected with a cyst has been evaluated (see Fig. 9). This affected kidney is from the first pig and has been analyzed $3.75 \mathrm{~h}$ after organ collection with an organ core temperature of $T_{\text {core }}=37.0^{\circ} \mathrm{C}$. Compared to the healthy kidney tissue of the first pig [cf., Fig. 9(a)], the spectral behavior of the cyst is completely different [cf., Fig. 9(b)], as a cyst contains a hydrous solution with cell components as well as hormones and electrolytes but almost no blood. This highly affects the spectral behavior. Between $\lambda=425$ to $900 \mathrm{~nm}$, the spectrometer cyst data are in the range of $I_{\text {reflectance }}=0.35$. The local minimum at $\lambda=580 \mathrm{~nm}$ is visible in all camera setup data. Further, the local maxima at $\lambda=710 \mathrm{~nm}$ and $\lambda=815 \mathrm{~nm}$ as well as the local minimum at $\lambda=740 \mathrm{~nm}$ are formed by the pushbroom and the 41-bands setup. The NCC between the healthy kidney data and cyst data is 0.0385 , which shows no relevant spectral correlation between these two types. Compared to the ground truth spectral cyst data of the spectrometer, the intensities of spectral data of the pushbroom camera as well as the 41-bands setup are slightly reduced. According to the NCC, the spectral curves of the pushbroom camera (0.996) and the 41-bands setup (0.930) are in good correlation with the reference spectra. Further, both setups are almost overlapping and comparable to each other with NCC of 0.926 . The spectral intensity data of the $3 \times 3$-VIS are slightly increased compared to the spectrometer data but follow the spectral trend as well.

\subsection{Blood Measurements}

The blood has been included into the blood circuit after $t=6.75 \mathrm{~h}$ of storing on ice. The first blood measurement has started $t=7 \mathrm{~h}$ after storing. The temperatures of the blood sample during the measurements are presented in Table 2 . The averaged blood temperature during all measurements has been $T_{\text {core }}=37.1^{\circ} \mathrm{C}$. 


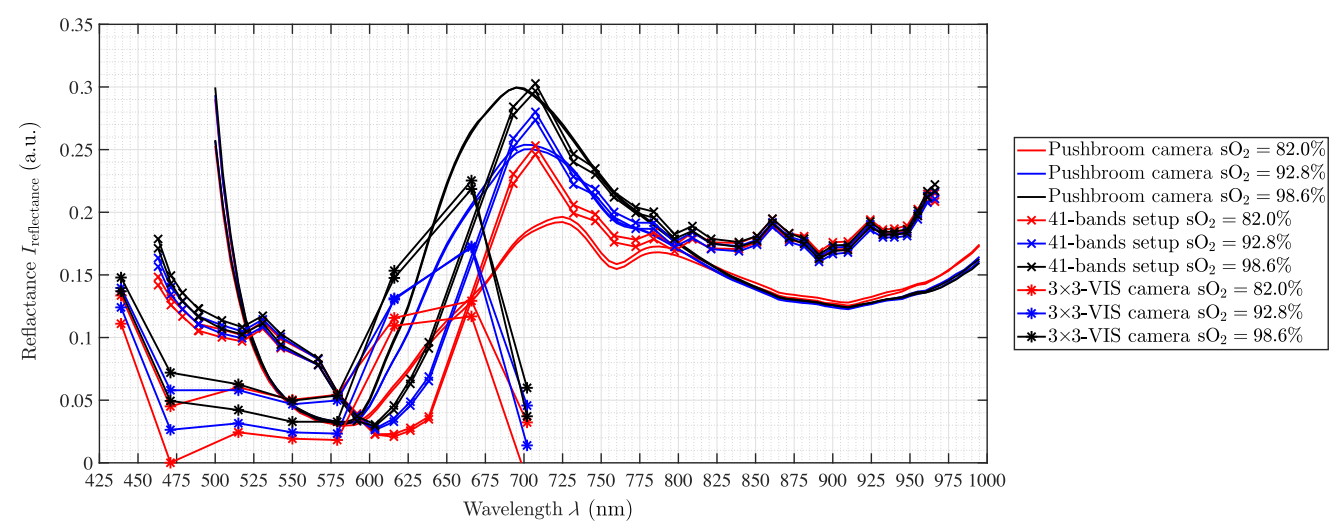

Fig. 10 Blood measurements of all three camera setups. For each setup, the two ROls average over the three measurements are presented.

The blood spectra show the expected behavior. There is a dependence on the degree of oxygenation of the blood with the reflectance characteristics. As shown in Fig. 10, the oxygenated blood curve has higher intensities in the near-IR ranging from $\lambda=600$ to $800 \mathrm{~nm}$ for the acquisition options of the hyperspectral pushbroom camera and the multispectral 41-bands setup. The multispectral $5 \times 5$-NIR data of the 41-bands setup show higher variability along the spectral curve compared to the hyperspectral pushbroom blood data as well as compared to the spectral trends in the $5 \times 5$-NIR organ measurements.

The individual behaviors for the different oxygenation levels with the characterized local minimum at $\lambda=760 \mathrm{~nm}$ for blood with low oxygen saturation is clearly visible for the pushbroom camera data. The 41-bands data follow the same trend, but it is less pronounced due to only 41 bands. The $3 \times 3$-VIS camera data only cover the visual range and follow the expected blood spectra trend for the eight bands until $\lambda=675 \mathrm{~nm}$. The $\lambda=700 \mathrm{~nm}$ band is far too low in terms of reflectance intensity. For the sixth $(\lambda=615 \mathrm{~nm})$ and seventh band $(\lambda=665 \mathrm{~nm})$ of this camera, the differentiation between oxygenated and deoxygenated blood is visible. This has been also expected for the eighth band $(\lambda=700 \mathrm{~nm})$ but could not be proven from the data. No spectrometer data have been analyzed for the blood measurements as the spectrometer data have shown total reflections, which omit the spectral behavior of the different blood samples.

\section{Discussion}

This study compares different spectral cameras for a possible clinical use, such as organs surveillance during transplantation by monitoring organ and blood specific spectral features. The performance of the camera systems has been evaluated using a reference spectrometer, which is validated using a standardized ColorChecker chart. The measured spectrometer data fit to the validation data with deviations up to about $5 \%$ due to the fact that the validation data are measured data by other authors. ${ }^{34,35}$ With respect to published as well as own measurement deviations, the data fit well. Further, the calculated Pearson coefficient represents a very high correlation. Thus the spectrometer data are used as reference system in this study.

In comparison with the reference system, two of the three camera setups show good results with regard to the reproduction of spectral information. These results could be obtained for measurement objects of different application areas: (1) standardized non-biological material and (2) complex biological materials. Both, in the analysis of the ColorChecker as well as the organs, the hyperspectral pushbroom camera (0.999 and 0.996, respectively) and the multispectral 41-bands setup (0.979 as well as 0.979$)$ show a very high positive correlation with the ground truth data of the spectrometer. The results for complex biological material, i.e., different organs, in the visible and NIR wavelength range, i.e., $\lambda=515$ to $995 \mathrm{~nm}$ for the pushbroom camera and $\lambda=470$ to $960 \mathrm{~nm}$ for the 41-band setup, are valid compared with published tissue spectra. ${ }^{37}$ The measured and calculated reflectance in the stated $\lambda$-ranges reproduces the real optical behavior and can show relevant tissue characteristics as $\mathrm{Hb}$ - and water-specific properties. 
This applies for the pushbroom camera as well as for both cameras of the 41-band setup. The better reproducibility of the specific reflectance for the pushbroom setup is caused by the higher spectral resolution.

In terms of reconstruction of the reflectance, the $3 \times 3$-VIS camera is comparable to the $4 \times 4$-VIS camera of the 41 -band setup but with less $\lambda$-bands. It becomes apparent that eight wavelength bands are not sufficient for a synchronized and comprehensive supervision of all specific organ markers such as $\mathrm{HbO}$ or water bands. This is manifested by the inaccurate representation of relevant peaks of the tissue chromophores, e.g., it will not become possible to detect relevant peak differences between $\mathrm{Hb}$ and $\mathrm{HbO}_{2}$ or other possible shifts in characteristic $\mathrm{Q}$ bands of present porphyrin complexes. For lung imaging, a double peak at about $\lambda=555 \mathrm{~nm}$ in the data shows the presence of $\mathrm{HbO}_{2}$ (see Fig. 8). Only in the $3 \times 3$-camera data, this double peak is not visible due to the few spectral bands. The multispectral $4 \times 4$-VIS camera holds 16 bands in a smaller wavelength interval as the multispectral $3 \times 3$-VIS camera, which at least are needed to detect small specific changes in the spectral appearance.

However, the $3 \times 3$-camera data behave similar to the $4 \times 4$-camera and pushbroom camera data for the first six wavelength bands, which are too few bands for wide spectral analysis. The last two bands at $\lambda=665 \mathrm{~nm}$ and $\lambda=700 \mathrm{~nm}$ always underestimate the reflectance intensity. It is not clear for what reason these two bands produce such false values. One reason could be a faulty correction matrix, which need further inspection. Another reason for that behavior could be due the lower transmission spectrum for that bands, which results in a lower signal-to-noise ratio (SNR) of these bands compared to the other bands. Such a low SNR also appears for the first data points of the hyperspectral pushbroom camera $(\lambda \approx 500$ to $515 \mathrm{~nm})$ and the first two spectral bands of the multispectral $4 \times 4$-VIS camera. However, the low SNR of the first data points of the hyperspectral pushbroom as well as the multispectral $4 \times 4$-VIS camera are caused by the low illumination intensity in that spectral interval due to the quartz-tungsten-halogen spectrum..$^{10,14,29}$

Despite the fact that the 41-bands setup has a lower spectral resolution than the pushbroom camera, both camera setups are suitable for identifying different physiological tissue properties like blood or other markers in the analyzed tissue sample or unphysiological organ structures like cysts on the kidney [cf., Fig. 9]. The higher spectral resolution of the pushbroom camera is at the cost of a longer recording time $t_{\text {record }}=6 \mathrm{~s}$, whereas the multispectral cameras allow real-time imaging. Although this study has been recorded with $20 \mathrm{fps}$, it is readily possible to use the study's setup with higher frame rate (e.g., 25 or $60 \mathrm{fps}$ ) and lower exposure settings. The reduced spatial resolution of multispectral snapshot cameras can be compensated using a suitable demosaicing algorithm. ${ }^{38}$

For the 41-bands setup data and the pushbroom camera data, it is visible that only the healthy kidneys have been purged of blood. The organs brain, heart, and lung show the local minimum at about $\lambda=760 \mathrm{~nm}$, which indicates the presence of blood. The kidney cyst data show the presence of blood as well. The varying expression of this local minimum indicates a different blood content in the analyzed organs. All setups can show the spectral variability of various organ types, which are caused by the different chemical and morphological compositions. For example, the expected reduction of reflectance by $50 \%$ for the heart compared to the other organs is clearly presented by all setups, as muscle tissue holds more chromophore than other tissue resulting in stronger absorbance. ${ }^{39}$.

The variation behavior of different reflectance intensity levels for different samples of the same type of organ or different ROIs within one organ sample is expected, as the reflectance intensity is also affected by the WD as well as illumination distance. Normally, this effect is compensated using the correction pipeline as proposed by Wisotzky et al. ${ }^{10}$ and Eq. (1). This includes the white reference image $I_{\text {white }}$, which is acquired using a flat calibration board, whereas the organs show a topological 3D structure causing deviations of the illumination and WD over the whole organ structure. Further, different organs of the same type are always of a slightly different anatomical texture and thus show small variations in spectral behavior. Therefore, the reconstructed reflectance intensity is dependent on the different organ samples as well as on the selected ROI (see Figs. 6 and 7). This difference is clearly visible in Fig. 7 as a larger gap between the 41-bands setup data and the pushbroom camera data, due to different specular reflections in the images and therefore different selected ROIs. 
Another reason for differences in reflectance intensity can be variant tissue temperature. The occurring temperature differences arising in this study are caused by different types of tissue, different densities, unequal organ weights, and sizes but do not significantly effect the results. We have started analyzing the organs of animal three and finished with animal one. This means, the time the organs are stored on ice has been in the range of $1 \mathrm{~h}$ for the brain of animal three up to $3.5 \mathrm{~h}$ for the heart of animal one. This temporal difference could affect the reflectance properties of each organ and tissue, ${ }^{40-42}$ which could explain the small discrepancies between the same organ types of different pigs. However, this aspect has not been the subject of this study and has not been analyzed in detail.

In general, both acquisition methods, pushbroom and snapshot setups, allow an exact reproduction of the physiological markers and pathological conditions of the analyzed anatomical structures. The precision of these representations is dependent of each setup, meaning the choice of the optimal setup would be dependent on the specific clinical aim. Different tissue parameters such as oxygen saturation, water, or blood concentration could be derived using all setups, whereas a higher number of spectral bands is preferable for a snapshot setup. Thus both basic acquisition principles could be feasible for clinical tissue analysis and differentiation or organ surveillance, e.g., to differentiate between single tissue structures as kidney, lung, or muscle, as well as to differentiate between healthy and pathological areas within one tissue type or organ.

To further verify the validity and reliability of the results obtained in this study, experiments on other biological objects or other organs of origin, i.e., other animal organs such as those from cattle or actual human tissue, would be useful.

\section{Conclusions}

In this work, we have presented and analyzed the possible use of three different spectral acquisition methods for intraoperative organ surveillance. All camera setups are able to reconstruct the spectral behavior of the analyzed organs in its available wavelength range. For accurate and robust analysis of clinically relevant biological materials, fine scanning of the analyzed spectral range is essential. Therefore, the hyperspectral pushbroom camera is the most accurate having spectral resolution of $5 \mathrm{~nm}$. However, with a minimum of $6 \mathrm{~s}$ recording time, it does not allow real-time monitoring. The setup with two multispectral $4 \times 4$-VIS and $5 \times 5$-NIR cameras represents a good compromise. It scans the wavelength range with 41 bands efficiently with a spectral resolution of $\sim 12.5 \mathrm{~nm}$ and allows real-time imaging as well as accurate analysis of the VIS-NIR range. Thus several tissue chromophores and indices can be analyzed in the range of 400 to $1000 \mathrm{~nm}$ to classify the tissue and its quality. Disadvantages of this camera setup is the small spectral gap at $\lambda=675 \pm 20 \mathrm{~nm}$ and the requirement of two cameras. The multispectral $3 \times 3$-VIS allows a better spatial resolution of the data but has the lowest number of bands. As a result, spectra cannot be reconstructed properly and physiological or pathological changes cannot be detected robustly.

Altogether, knowledge of the suitability of spectral camera systems to determine the exact chemical properties of a biological object could offer a promising opportunity to measure organor blood-specific parameters. In combination with previously published literature about optical organ and tissue behaviors, ${ }^{37,39,43-47}$ this study allows the selection of the optimal HMSI system for the use for a specific medical applications. ${ }^{12,31,48,49}$

\section{Disclosures}

The authors declare no conflicts of interest. The funders had no role in the design of the study; in the collection, analysis, or interpretation of data; in the writing of the manuscript; or in the decision to publish the results.

\section{Acknowledgments}

The authors highly appreciate the support of Prof. Eike Brunner, Chair of Bioanalytical Chemistry, TU Dresden, Germany. This research was funded by the German Federal 
Ministry of Education and Research under Grant No. 16SV8061 in the project MultiARC. Further, it was funded by the European Social Fund and co-financed by tax funds based on the budget approved by the members of the Saxon State Parliament.

\section{References}

1. G. M. Abouna, "Organ shortage crisis: problems and possible solutions," Transplant. Proc. 40(1),34-38 (2008).

2. Deutsche Stiftung Organtransplantation, "Jahresbericht: Organspende und Transplantation in Deutschland 2019," https://dso.de/SiteCollectionDocuments/DSO-Jahresbericht\%202019.pdf (2020).

3. Deutsche Stiftung Organtransplantation, "Jahresbericht: Organspende und Transplantation in Deutschland 2018," https://dso.de/SiteCollectionDocuments/DSO-Jahresbericht\%202018.pdf (2019).

4. S. Mittal et al., "A re-evaluation of discarded deceased donor kidneys in the UK: are usable organs still being discarded?" Transplantation 101(7), 1698-1703 (2017).

5. S. A. Husain et al., "Characteristics and performance of unilateral kidney transplants from deceased donors," Clin. J. Am. Soc. Nephrol. 13(1), 118-127 (2018).

6. S. Hosgood et al., "Normothermic machine perfusion for the assessment and transplantation of declined human kidneys from donation after circulatory death donors," Br. J. Surg. 105(4), 388 (2018).

7. S. Hosgood et al., "Successful transplantation of human kidneys deemed untransplantable but resuscitated by ex vivo normothermic machine perfusion," Am. J. Transplant. 16(11), 3282-3285 (2016).

8. "Nierentransplantation 2018-Grafiken zum Tätigkeitsbericht 2018" (2019).

9. G. Lu and B. Fei, "Medical hyperspectral imaging: a review," J. Biomed. Opt. 19(1), 010901 (2014).

10. E. L. Wisotzky et al., "Validation of two techniques for intraoperative hyperspectral human tissue determination," J. Med. Imaging 7(6), 065001 (2020).

11. N. T. Clancy et al., "Surgical spectral imaging," Med. Image Anal. 63, 101699 (2020).

12. A. Holmer et al., "Hyperspectral imaging in perfusion and wound diagnostics-methods and algorithms for the determination of tissue parameters," Biomed. Eng./Biomed. Tech. 63(5), 547-556 (2018).

13. E. L. Wisotzky et al., "Intraoperative hyperspectral determination of human tissue properties," J. Biomed. Opt. 23(9), 091409 (2018).

14. R. Mühle et al., "Workflow and hardware for intraoperative hyperspectral data acquisition in neurosurgery," Biomed. Eng./Biomed. Tech. 66(1), 31-42 (2021).

15. S. L. Best et al., "Renal oxygenation measurement during partial nephrectomy using hyperspectral imaging may predict acute postoperative renal function," J. Endourol. 27(8), 10371040 (2013).

16. F. Tetschke et al., "Hyperspectral imaging for monitoring oxygen saturation levels during normothermic kidney perfusion," J. Sens. Sens. Syst. 5(2), 313-318 (2016).

17. A. Holmer et al., "Oxygenation and perfusion monitoring with a hyperspectral camera system for chemical based tissue analysis of skin and organs," Physiol. Meas. 37(11), 2064 (2016).

18. W. Markgraf et al., "Algorithm for mapping kidney tissue water content during normothermic machine perfusion using hyperspectral imaging," Algorithms 13(11), 289 (2020).

19. B. Kossack et al., "Local blood flow analysis and visualization from RGB-video sequences," Curr. Directions Biomed. Eng. 5(1), 373-376 (2019).

20. B. Kossack et al., "Local remote photoplethysmography signal analysis for application in presentation attack detection," in Vision, Modeling and Visualization, H.-J. Schulz, M. Teschner, and M. Wimmer, Eds., The Eurographics Association (2019).

21. N. T. Clancy et al., "Multispectral imaging of organ viability during uterine transplantation surgery," Proc. SPIE 8935, 893510 (2014).

22. N. T. Clancy et al., "Multispectral imaging of organ viability during uterine transplantation surgery in rabbits and sheep," J. Biomed. Opt. 21(10), 106006 (2016). 
23. W. Markgraf et al., "Algorithms for mapping kidney tissue oxygenation during normothermic machine perfusion using hyperspectral imaging," Biomed. Eng./Biomed. Tech. 63(5), 557-566 (2018).

24. W. Markgraf et al., "Hyperspectral imaging for ex-vivo organ characterization during normothermic machine perfusion," Eur. Urol. Suppl. 17(2), e767 (2018).

25. J. J. Workman and L. Weyer, Practical Guide to Interpretive Near-Infrared Spectroscopy, 1st ed., CRC Press, Boca Raton, Florida (2007).

26. P. Singh et al., "Renal oxygenation and haemodynamics in acute kidney injury and chronic kidney disease," Clin. Exp. Pharmacol. Physiol. 40(2), 138-147 (2013).

27. C. Jimenez et al., "Ultrasonography in kidney transplantation: values and new developments," Transplant. Rev. 23(4), 209-213 (2009).

28. S. J. Galgano et al., "Optimizing renal transplant Doppler ultrasound," Abdominal Radiol. 43(10), 2564-2573 (2018).

29. E. L. Wisotzky et al., "Validation of two techniques for intraoperative hyperspectral human tissue determination," Proc. SPIE 10951, $109511 \mathrm{Z}$ (2019).

30. M. Allan et al., "Stereo correspondence and reconstruction of endoscopic data challenge," (2021).

31. E. L. Wisotzky et al., "Surgical guidance for removal of cholesteatoma using a multispectral 3D-endoscope," Sensors 20(18), 5334 (2020).

32. J. C. Rosenthal and P. Eisert, "Remote visual inspection, a real-time image-based stereo video processing chain for 3D endoscopy," Inspect Int. -World Vision 2019(2), 48-50 (2019).

33. F. Zilly et al., "The stereoscopic analyzer—an image-based assistance tool for stereo shooting and 3D production," in IEEE Int. Conf. Image Process., IEEE, pp. 4029-4032 (2010).

34. C. S. McCamy et al., "A color-rendition chart," J. Appl. Photogr. Eng. 2(3), 95-99 (1976).

35. D. Pascale, RGB Coordinates of the Macbeth Colorchecker, Vol. 6, The BabelColor Company (2006).

36. R. Giovannetti, "The use of spectrophotometry UV-VIS for the study of porphyrins, Chapter 6," Macro Nano Spectrosc. 1, 87-108 (2012).

37. S. L. Jacques, "Optical properties of biological tissues: a review," Phys. Med. Biol. 58(11), R37-R61 (2013).

38. S. Mihoubi et al., "Illumination-robust multispectral demosaicing," in Int. Conf. Image Process. Theory, Tools Appl. (IPTA), Vol. 7, pp. 1-6 (2017).

39. E. L. Wisotzky et al., "Determination of optical properties of human tissues obtained from parotidectomy in the spectral range of 250 to $800 \mathrm{~nm}$, J. Biomed. Opt. 24(12), 125001 (2019).

40. J. Laufer et al., "Effect of temperature on the optical properties of ex vivo human dermis and subdermis," Phys. Med. Biol. 43, 2479-2489 (1998).

41. W. Markgraf et al., "Hyperspectral imaging of porcine kidneys during normothermic ex vivo perfusion: an analysis of tissue-related renal ischemia injury," Transplant Int. 33(Suppl. 2), 35 (2020).

42. S. M. Jaywant et al., "Temperature-dependent changes in the optical absorption and scattering spectra of tissues: correlation with ultrastructure," Proc. SPIE 1882, 218-229 (1993).

43. W.-F. Cheong, S. A. Prahl, and A. J. Welch, "A review of the optical properties of biological tissues," IEEE J. Quantum Electron. 26(12), 2166-2185 (1990).

44. E. L. Wisotzky et al., "Determination of optical properties of cholesteatoma in the spectral range of 250 to $800 \mathrm{~nm}$," Biomed. Opt. Express 11(3), 1489-1500 (2020).

45. M. Friebel et al., "Determination of optical properties of human blood in the spectral range 250 to $1100 \mathrm{~nm}$ using Monte Carlo simulations with hematocrit-dependent effective scattering phase functions," J. Biomed. Opt. 11(3), 034021 (2006).

46. E. V. Salomatina et al., "Optical properties of normal and cancerous human skin in the visible and near-infrared spectral range," J. Biomed. Opt. 11(6), 064026 (2006).

47. M. Friebel et al., "Influence of oxygen saturation on the optical scattering properties of human red blood cells in the spectral range 250 to $2000 \mathrm{~nm}$," J. Biomed. Opt. 14(3), 034001 (2009). 
48. E. L. Wisotzky et al., "A multispectral 3D-endoscope for cholesteatoma removal," Curr. Directions Biomed. Eng. 6(3), 257-260 (2020).

49. G. Florimbi et al., "Towards real-time computing of intraoperative hyperspectral imaging for brain cancer detection using multi-GPU platforms," IEEE Access 8, 8485-8501 (2020).

Richard Mühle studied biomedical engineering at Technische Universität Ilmenau in 2008. With the end of a research stay at Universiti Teknologi Malaysia with focus on design of spinal implants, he completed his master's degree at Ilmenau 2015. In the last years, he worked as a research assistant at Technical University Dresden on several projects with topics relating to tumor detection during brain surgery and normothermic transplantation technology.

Wenke Markgraf is currently pursuing a doctoral degree at the Chair of Bioanalytical Chemistry at the Technische Universität Dresden (TUD). Together with the Institute of Biomedical Engineering at the TUD, she investigates strategies for the objective functional assessment of ex vivo organs. The focus of her work ranges from biochemical and histological analysis to applications of hyperspectral imaging, image processing, and chemometrics.

Anna Hilsmann heads the Vision and Imaging Technologies Department and the Computer Vision \& Graphics Group at the Fraunhofer HHI, Berlin, Germany. She received her Dipl.-Ing. degree in electrical engineering in 2006 and her Dr.-Ing. degree "with highest honors" from Humboldt University Berlin in 2014. Her research interests include computer vision and computer graphics, especially image and video analysis, as well as scene understanding and modelling, in application areas like medicine, multimedia, and security.

Hagen Malberg: Biography is not available.

Peter Eisert received his Dr.-Ing. degree in 2000 from the University Erlangen and worked as a postdoctoral fellow at Stanford University. He is a professor for visual computing at Humboldt University Berlin and heading the Vision and Imaging Technologies Department at Fraunhofer HHI, Berlin, Germany. He is coordinating and initiating numerous research projects on image/ video analysis and synthesis, face processing, computer vision and graphics in multimedia, security, and medical applications. He has published more than 150 conference and journal papers and is an associate editor of JIVP and JVCI.

Eric L. Wisotzky received his BSc and MSc degrees from Heidelberg University, Heidelberg, Germany, in 2012 and 2014, respectively. After a research visit at Sydney Medical School of Sydney University, he is currently working toward his doctoral degree at Humboldt University of Berlin and Fraunhofer Heinrich-Hertz-Institute. Together with the Department of Otolaryngology of Charité-Universitätsmedizin Berlin, he is working on new image-guided surgery techniques. His research interests span from imaging physics to image processing and modeling in medicine. $\mathrm{He}$ is a member of SPIE. 